\title{
Maximal-entropy initial state of the Universe as a microscopic description of inflation
}

\author{
Ram Brustein ${ }^{1,2, *}$ and A. J. M. Medved ${ }^{3,4, \dagger}$ \\ ${ }^{1}$ Department of Physics, Ben-Gurion University, Beer-Sheva 84105, Israel \\ ${ }^{2}$ Theoretical Physics Department, CERN, 1211 Geneva 23, Switzerland \\ ${ }^{3}$ Department of Physics \& Electronics, Rhodes University, Grahamstown 6140, South Africa \\ ${ }^{4}$ National Institute for Theoretical Physics (NITheP), Western Cape 7602, South Africa
}

(Received 6 January 2020; accepted 21 May 2020; published 2 June 2020)

\begin{abstract}
We propose that the initial state of the Universe was an isotropic state of maximal entropy. Such a state can be described in terms of a state of closed, interacting, fundamental strings in their high-temperature Hagedorn phase, which constitutes a novel microscopic model for the state of the Universe when it is at the highest sustainable temperature. This state resolves the big-bang singularity by replacing the past of the hot big-bang Universe and sets inflationary initial conditions for the subsequent evolution of the thermal radiation and the semiclassical cosmological geometry. The entropy density in this state is equal to the square root of the energy density in Planck units, while the pressure is positive and equal to the energy density. These relations imply a maximally large entropy density and, therefore, a state that cannot be described by a semiclassical spacetime geometry. If one nevertheless insists on an effective semiclassical description of this state, she can do so by ignoring the entropy. This leads to a partially equivalent description in which the pressure appears to be negative and equal in magnitude to the energy density, as if the energy-momentum tensor was that of a cosmological constant. From this effective perspective, the state describes a period of string-scale inflation of minimal duration. The stringy state ultimately decays, possibly by a process akin to Hawking radiation, and undergoes a transition into a phase of hot radiation. But, from the effective perspective, the same decay corresponds to the heating of the Universe at the end of inflation. Small quantum mechanical fluctuations in the initial state lead to a scale-invariant temperature anisotropies in the hot radiation. The temperature anisotropies are interpreted in the effective description as arising from quantum fluctuations of the curvature and an effective inflaton field. The stringy microscopic description determines the parameters of the model of inflation, as well as the cosmological observables, in terms of the string length scale and coupling strength. In particular, it describes a high-scale model of inflation with a large scalar-to-tensor ratio which is qualitatively compatible with the cosmological observations. Our framework is similar, conceptually, to a recent description of black holes in terms of a maximal entropy state of strings in the Hagedorn phase.
\end{abstract}

DOI: 10.1103/PhysRevD.101.123502

\section{INTRODUCTION}

The hot big-bang model provides an accurate description of the cosmological evolution of our Universe starting from a thermal state of hot radiation. However, the same model also faces some unresolved issues that are related to properties of the initial state: the large-scale smoothness, the small-scale inhomogeneities, and the smallness of the

\footnotetext{
ramyb@bgu.ac.il

j.medved@ru.ac.za
}

Published by the American Physical Society under the terms of the Creative Commons Attribution 4.0 International license. Further distribution of this work must maintain attribution to the author(s) and the published article's title, journal citation, and DOI. Funded by SCOAP. spatial curvature. As a way to extend the validity of the hot big-bang model and resolve some of its shortcomings, it was proposed that, prior to the thermal phase, the Universe expanded exponentially during a relatively long period of cosmic inflation. The Universe then "reheated" in an event that marks the beginning of the thermal phase; this being the essence of the inflationary paradigm $[1,2]$.

This paradigm provides a framework for an effective description of the period of exponential expansion. The scale of inflation, its duration, and the type of matter that drives it-the so-called inflaton field-are all undetermined and subject to rather weak constraints. For any given model of inflation, these parameters need to be specified in a way that predicts the cosmological observables; in particular, the spectral properties of the observed temperature inhomogeneities. Many of the 
puzzles surrounding the inflationary paradigm are associated with attempts to identify the inflaton field with one of the fields in the standard model or in some type of grand unified theory or in some version of string theory.

The inflationary paradigm has been criticized recently [3,4] (see also [5]) on the grounds that a weakly coupled model of inflation is not self-consistent when implemented within semiclassical gravity. More specifically, the selfreproduction property of such models leads to scenarios of eternal inflation which imply that semiclassical evolution breaks down. Furthermore, the scale of inflation that is needed to reproduce the correct spectra of perturbations must be much lower than the natural cutoff of the theory, the Planck scale.

Meanwhile, the problem of an initial singularity continues to persist in the inflationary paradigm. This concern is well known [6,7] but sometimes dismissed by arguing that the singularity decouples from horizon-scale physics. The premise being that cosmological observables, which are determined solely by the latter, will not be affected by whatever does resolve the singularity. However, this is not a truly admissible argument because, in the words of Hawking [8], "The only way to have a scientific theory is if the laws of physics hold everywhere including at the beginning of the Universe." Some remnants of the problem linger and reappear when one attempts to define a measure on the space of initial conditions. Just like the analogous problem for a black hole $(\mathrm{BH})$ [9], it turns out that the resolution is much more surprising than a small tweak which transforms the singularity into an epoch of large but finite curvature.

Here, we propose a microscopic model for the state of the Universe when it is at the highest sustainable temperature for a state of strings, the Hagedorn temperature. This state replaces the past of the hot big-bang Universe, resolves the singularity, and provides the initial conditions for the subsequent evolution of the thermal radiation and the semiclassical cosmological geometry.

Our microscopic model is guided, in large part, by the polymer model of BHs $[10,11]$, which suggests that a maximally large entropy is an essential feature of nonsingular gravitational states. Maximal entropy in this context means the saturation of the causal entropy bound $[12,13]$; then the entropy density $s$ is as large as it can be in relation to the energy density $\rho$ in appropriate units. Fundamental, closed strings in the Hagedorn phase are in just such a maximally entropic state. Just like for BHs, the microscopic description of cosmology in terms of this hot string state is nonsingular. In both cases, the apparent singularity is resolved by making dramatic changes to the state on horizon-sized scales. However, whereas the changes for a $\mathrm{BH}$ are to its interior region, it is the prehistory of the thermal state that is changed in the cosmological picture. Whether one is talking about BHs or cosmology, the cost of regularization is that the hot string state cannot be described by a semiclassical geometry.

Our proposal differs from previous ones that involved the Hagedorn phase of strings, such as string gas cosmology [14-18]. In spite of the seemingly similar context, the actual meanings of the "Hagedorn phase" in our model and in the scenario of string gas cosmology are quite different. In string gas cosmology, the strings are at a temperature below the Hagedorn temperature and need some compact spatial dimensions to wind around. Furthermore, the number of large spatial dimensions is at most three. String interactions are not essential for this scenario. For our model, in contrast, the temperature is above the Hagedorn temperature, there is no need for winding modes around spatial dimensions and interactions are essential. These differences affect the mechanism of exit from inflation, as well as the cosmological perturbations and thus the predictions for cosmological observables.

Our proposal also differs from previous ones suggesting that the initial state of the Universe is a maximal entropy state as in holographic cosmology [19,20]. Again, in spite of the seemingly similar context, the details are quite different. There are indeed some similarities between the two models in that both involve a $p=\rho$ fluid and so one of maximal entropy density. However, our basic claim is that such a fluid, which saturates the causal entropy bound, could not possibly be described in terms of semiclassical physics. Furthermore, if one does insist on a semiclassical description, then it should be described in terms of a dual $p=-\rho$ fluid. This can be contrasted with the black hole fluid of holographic cosmology, for which each constituent is itself a solution of Einstein's equation. Additionally, holographic cosmology calls for a semiclassical evolution between a $p=\rho$ phase toward a $p=-\rho$ phase such that inflation corresponds to the $p=-\rho$ phase. This distinction leads to a difference in predictions for cosmological observables. The most pronounced difference is in the prediction for the tensor to scalar ratio, which in holographic cosmology has a parametrically small value.

Even if lacking a geometry, a state of hot, interacting, closed strings can, as discussed in [10], be described by a simple free energy which is expressible as a power series in the entropy density. For the BH polymer, $s$ and the other thermodynamic densities had to have nontrivial radial profiles. In the context of cosmology, however, approximate isotropy and homogeneity are now mandatory features; meaning that the free energy and all of its associated thermodynamic densities are approximately constant in space and time.

Although the current proposal is conceptually similar to our proposed resolution of the BH singularity [9], it does differ in some important ways that go beyond questions about spacetime (in)dependence. For instance, who plays the role of the "score-keeping observer"? In the case of $\mathrm{BHs}$, it is clear that the asymptotic external observer serves 
this purpose. The cosmological analogue-perhaps not quite as obvious-is the late-time or "FriedmannRobertson-Walker (FRW) observer." This is because the past of this observer, before the beginning of the hotradiation phase, is the analogue of the interior of the $\mathrm{BH}$. As similarly argued for the BH case in [21], all proposals for the prehistory are perfectly acceptable as long as they are self-consistent, able to reproduce the observable Universe and compatible with the laws of physics (see also [22]). From the microscopic point of view, the puzzles of the FRW observer originate from trying to explain the initial quantum state using effective semiclassical terms. The same situation was prevalent for $\mathrm{BHs}$ and led to their infamous paradoxes. As will be shown here, the FRW observer can (and usually does) interpret our maximally entropic state as one of vanishing entropy (and/or temperature) and approximately describes it by using the flatspace slicing of a de Sitter (dS) spacetime.

In our cosmological model, a geometric description of the past may be absent, but one can still adopt the equivalent representation of gravity as an inertial effect in a conformally flat space. ${ }^{1}$ Conformally flat spacetime coordinates, $t_{s t}, r_{s t}$, etc., would then represent labels for the position of the strings but physical observables will not depend on these fiducial coordinates. From this point of view, gravity is an emergent effect; it is a long-distance description of the microscopic forces between constituents. It is only when gravity is semiclassical that both of the descriptions, geometric and inertial, can coexist.

In the microscopic model, the scale of inflation is fixed by the string scale and the duration of the exponential expansion, as perceived by the FRW observer, is the logarithm of the entropy of the Universe in natural units. In this sense, inflation is as short as it can be. But the maximally entropic state still needs to be large enough to describe a large Universe, so that one could also try to understand how the initial state came to be so large. We will defer this issue to a future investigation but still want to suggest some possibilities. For example, the Universe could start out in a large, weakly curved, contracting phase for which it was initially large and empty-as in pre-big-bang scenarios [23] or ekpyrotic models [24] —and then undergo a phase of contraction in which the number of Hubble-sized regions grows and the strings eventually heat up to the Hagedorn temperature. This would be the analogue of a large matter system collapsing to form a BH [25]. However, it is not important for current purposes to track the prehistory of the initial state. A good choice of an initial state is always a part of a good description of any physical system.

\footnotetext{
${ }^{1}$ The choice of conformally flat rather than just flat is to accommodate the constancy of the thermodynamic densities as discussed above. This point is elaborated on in Subsection II A.
}

Throughout the paper, we typically focus on the case of $D=d+1=3+1$ spacetime dimensions. However, our conclusions are unchanged for $D>4$.

\section{INITIAL STATE OF THE UNIVERSE}

We propose to replace the semiclassical, singular past of the hot big-bang Universe by a quantum epoch of large but finite curvature. The quantum state defines the initial state of what evolves into a semiclassical, asymptotically dS Universe.

In the next subsection, we describe the initial state as a thermal state of closed, interacting, fundamental strings at a temperature which is just above but parametrically close to the Hagedorn temperature, $\left(T-T_{\mathrm{Hag}}\right) / T_{\mathrm{Hag}}=\epsilon \ll 1$. The equation of state of the strings is $p=\rho$, which consequently implies an entropy density of $s=\sqrt{\rho}$ in Planck units. The total entropy of the state is also specified and the free energy that describes the state is given explicitly in Eq. (1). Thus, what is presented is a complete description of the initial state for given values of the total entropy $S$ (or equivalently, total volume $V$ ), the Hagedorn temperature $T_{\text {Hag }}$, the small parameter $\epsilon$ and the string coupling $g_{s}^{2}$.

Then, in Sec. II B, we explain how the FRW observer can interpret the maximally entropic state as one of vanishing entropy (and/or temperature) and approximately describe it by using the flat-space slicing of an asymptotically $\mathrm{dS}$ spacetime. From this point of view, the FRW observer invents an effective history of the Universe: the inflationary paradigm. We emphasize that physical observables are the results of the measurements that are made by an FRW observer after the end of inflation. The FRW observer can only measure a portion of the postinflationary Universe at finite times. However, the whole state does become observable as $t \rightarrow \infty$. We also emphasize that, if the observables can be predicted correctly, this is all there is to it. The rest of the theory consists of conceptualizations that can never be verified and must forever remain ambiguous.

In Sec. II C, we show how it is possible to reconcile the two points of view about the history of the early Universe, hot strings and inflationary dS, as both lead to the same picture of semiclassical evolution at late times.

\section{A. Microscopic perspective: Hagedorn phase of fundamental strings}

Closed strings in the Hagedorn phase have an exponentially large density of states (e.g., [26-28]) and are, therefore, particularly well suited for describing states with high entropy. Moreover, the equation of state connecting the pressure $p$ and the energy density $\rho$ for such hightemperature string theory is known to be $p=\rho$. And so $p$ is as large as a single-fluid pressure could be while still respecting causality. The standard thermodynamic relation $\rho+p=s T$ for the energy density, pressure, entropy 
density $s$, and temperature $T$ then leads to $s=\sqrt{\rho}$ in Planck units, from which one obtains $1 / T=d s / d \rho=s / 2 \rho$ or $s T=2 \rho$. Meaning that $s$ is also as large as it could ever be in comparison to $\rho$.

An important consequence of the maximal entropy and pressure is that these require exact spatial flatness and the vanishing of the cosmological constant. This is because the introduction of any such sources would reduce the ratio $p / \rho$ away from unity.

We now briefly review, for completeness, relevant parts of the discussion in $[10,11]$ and then adapt them to the current cosmological setup.

Both the energy $E$ and entropy $S$ of noninteracting, closed strings in the Hagedorn phase scale linearly with the total length of string $L=N l_{s}$, so that $S \sim N$ and $E \sim N / l_{s}$ ( $l_{s}$ is the string length scale). The free energy $F=E-S T$ vanishes for noninteracting strings at the Hagedorn temperature $T_{\mathrm{Hag}}=M_{s} /(4 \pi) \quad\left(M_{s}=1 / l_{s}\right.$ is the string mass $)$. Then, for temperatures $T$ close to $T_{\mathrm{Hag}}$, the free energy $F$ should be parametrically smaller than $N / l_{s}$. We will use a dimensionless parameter $\epsilon$ to parametrize this small number, $\epsilon=\left(T-T_{\mathrm{Hag}}\right) / T_{\mathrm{Hag}} \ll 1$. For the case of interacting strings, it makes sense to consider both positive and negative $\epsilon$; however, one can expect a phase transition when $\epsilon<0$ (see below).

Interactions between strings take place at their intersections. The strength of the interactions-that is, the probability that two different strings join to form one single longer loop or that a single string splits up into two shorter loops-is determined by the string coupling $g$ and is equal to $g^{2}$. For weakly coupled strings, $g^{2}=M_{s}^{2} / M_{P}^{2}$, where $M_{P}$ is the Planck mass. If $V$ is the volume of the region of space that is occupied by the strings (in terms of flat fiducial coordinates as explained in Sec. I), then the volume density of intersections is $N^{2} / V$, and so the leading-order effects of interactions can be described by including a term scaling as $g^{2} N^{2} / V=N\left(g^{2} N / V\right)$ in the free energy. Hence, the free energy for interacting strings in the Hagedorn phase is expressible in terms of the entropy density $s=N / V$ as follows [10,11]:

$$
-\left(\frac{F}{V T_{\mathrm{Hag}}}\right)_{\text {strings }}=\epsilon s-\frac{1}{2} g^{2} s^{2}+\cdots,
$$

where string units $\left(l_{s}=1\right)$ have now been adopted and the first term on the right follows directly from previous relations given that $\rho=E / V=N T_{\mathrm{Hag}} / V$. The ellipsis denotes higher-order interactions, which are small under the conditions that we consider, to be discussed in more detail later. Not coincidentally, this free energy is formally similar to those in the literature on interacting polymers (e.g., [29]).

Extremizing the free energy with respect to $s$, one finds that

$$
s=\frac{\epsilon}{g^{2}} \text {. }
$$

The energy density and pressure now follow from the standard thermodynamic definitions,

$$
\begin{aligned}
& \rho=\frac{1}{2} \frac{\epsilon^{2}}{g^{2}}, \\
& p=\frac{1}{2} \frac{\epsilon^{2}}{g^{2}},
\end{aligned}
$$

and so the equation of state of the associated fluid is indeed $p=\rho$. Comparing Eqs. (2) and (3), one obtains the similarly advertised form $s=2 \sqrt{\rho / g^{2}}$ or, more simply, $s \epsilon=2 \rho$. The latter suggests that $\epsilon$ acts like an effective temperature, not to be confused with the local temperature of the string state which is the Hagedorn temperature. Whereas the BH version of $\epsilon$ turned out to be the Hawking temperature, it will be (re)calculated later on and identified as the Gibbons-Hawking temperature [30].

Let us recall that, for the $\mathrm{BH}$ scenario, $\epsilon$ and thus the various densities have radial profiles. ${ }^{2}$ As the cosmological fluid has to be approximately isotropic and homogeneous, the densities $s$ and $\rho$, as well as the pressure $p$ and the effective temperature $\epsilon$ will all be regarded as constants. Although, near the boundary of the string state, there is likely to be some deviations from this constancy.

To specify the solution completely, $\epsilon$ needs to be fixed in a way that is independent of Eq. (2). For this, we will relate $\epsilon$ to the causal connection scale $R_{\mathrm{CC}}$ [12], which can, in general, be viewed as the relativistic analogue of the Jeans length. The notion of a Jeans length is applicable in the current context because of the fact that, at long distances, the string interactions are dominated by gravity $[25,31,32]$. For $\mathrm{BHs}, R_{\mathrm{CC}}$ corresponds to the Schwarzschild radius and thus serves as the linear scale for the whole string state. But the cosmological string state can encompass many causally disconnected regions; meaning that $R_{\mathrm{CC}}$ must be the linear scale as measured in fiducial coordinates of just one such region.

There are several ways in which $R_{\mathrm{CC}}$ can be estimated. It was originally estimated in [12] in its capacity as a "Jeanslike" length scale, which means considering the equation of motion for a generic perturbation. Because the string state is translation invariant, it is convenient if perturbations are expressed in their Fourier-space form $\delta_{k}$; in which case, the relevant equation is

$$
\ddot{\delta}_{k}+\left(k^{2}-R_{\mathrm{CC}}^{-2}\right) \delta_{k}=0,
$$

\footnotetext{
${ }^{2}$ This dependence was not always made explicit in previous papers.
} 
where $k=|\vec{k}|$ is the wave number and a dot represents a time derivative, both with respect to the fiducial coordinates. Following [12], one will find that

$$
R_{\mathrm{CC}}^{-2} \simeq g^{2} \operatorname{Max}[\rho / 3-p, \rho+p] .
$$

When $p=0$, the causal connection scale $R_{\mathrm{CC}}$ is exactly the Jeans length, $R_{\mathrm{CC}}^{-2}=g^{2} \rho$. For the current case of $p=\rho$, this is still approximately true,

$$
R_{\mathrm{CC}}^{-2}=2 g^{2} \rho=\frac{\epsilon^{2}}{l_{s}^{2}},
$$

where we have used Eqs. (3), (4) and restored the dependence on $l_{s}$. Notice that, for a cosmological setup, the first equality in Eq. (7) implies that $R_{\mathrm{CC}}$ is the Hubble radius $H^{-1}$ and then $\epsilon \sim R_{\mathrm{CC}}^{-1} \sim H$ is the Gibbons-Hawking temperature.

A different (but related) way to estimate $R_{\mathrm{CC}}$ is to note that, if the string interactions are indeed dominated by gravity, then the total interaction energy within a causally connected region should be parametrically equal to its gravitational energy. ${ }^{3}$ The energy of the string within such a region is given by

$$
E_{\text {string }} \simeq \rho R_{\mathrm{CC}}^{d} \simeq \frac{\epsilon^{2}}{g^{2}} R_{\mathrm{CC}}^{d}
$$

and the gravitational energy can be expressed using the Newtonian potential,

$$
E_{\text {grav }} \simeq g^{2} \frac{E_{\text {string }}^{2}}{R_{\mathrm{CC}}^{d-2}} \simeq \frac{\epsilon^{4}}{g^{2}} R_{\mathrm{CC}}^{d+2},
$$

along with the identification $G_{N}=l_{P}^{d-1}=g^{2} l_{s}^{d-1}=g^{2}$. Then, since the interaction energy should be the same order as the net energy of the string, it follows that

$$
\frac{\epsilon^{2}}{g^{2}} R_{\mathrm{CC}}^{d} \simeq \frac{\epsilon^{4}}{g^{2}} R_{\mathrm{CC}}^{d+2},
$$

the solution of which is, once again, $R_{\mathrm{CC}}^{-2}=\frac{\epsilon^{2}}{l_{s}^{2}}$.

Yet another way to arrive at Eq. (7) is to apply a double scaling limit in which the relative deviation of the string temperature from the Hagedorn temperature is tuned in response to the tuning of the total length of the string $N[10,11]$.

In a $(d=3)$ cosmological setup, for a yet unspecified external observer, we can interpret the above results as follows: The energy within a sphere of radius $R_{\mathrm{CC}}$ scales as

\footnotetext{
${ }^{3}$ Here, we are temporarily generalizing the number of spatial dimensions to $d \geq 3$ to highlight the fact that the scaling of $\epsilon$ and thus the various densities are independent of this number.
}

$E \sim R_{\mathrm{CC}}^{3} \epsilon^{2} / g^{2} \sim R_{\mathrm{CC}} / g^{2}$ and the entropy as $S \sim R_{\mathrm{CC}}^{3} \epsilon / g^{2} \sim$ $R_{\mathrm{CC}}^{2} / g^{2}$. So that, if we identify $R_{\mathrm{CC}}$ with the inverse of the Hubble radius $H^{-1}$ of some epoch of the Universe and use that $g$ is the inverse of the Planck mass $M_{P}$ in string units, then the energy and entropy within a Hubble volume go as

$$
E_{H} \sim \frac{M_{P}^{2}}{H}
$$

and

$$
S_{H} \sim \frac{M_{P}^{2}}{H^{2}}
$$

which agrees with the expected scaling relations of the energy within the horizon and the Gibbons-Hawking entropy, respectively. If $R_{\mathrm{CC}}=H^{-1}$ is large (in string units), the Hubble scale is smaller than the string scale because then $H \sim \epsilon M_{s} \ll M_{s}$.

We will be interested in situations in which not only $H^{-1}$ is large but the Universe is "large" in another sense; namely that its total entropy far exceeds the entropy in a single Hubble region, $S_{\text {tot }} \gg S_{H}$. There will then be a large number $n_{H}=V_{\text {tot }} / H^{-3}$ of causally disconnected regions and the entropy $S_{\text {tot }}=S_{H} n_{H}$ will saturate the causal entropy bound, $S \leq \sqrt{E V}[12,13]$.

Let us now discuss the symmetries of the string state. These will be essential for establishing the connection between the semiclassical perspective and the microscopic calculation of the correlation function for the perturbations. The string state is invariant in an obvious way under rotations and translations of the fiducial coordinates (except near the boundary of the state). A less obvious symmetry is one in which the fiducial coordinates are rescaled as $x_{i} \rightarrow \lambda x_{i}$, with $\lambda$ being some numerical constant. To understand the origin of this scaling symmetry, let us recall that, in a given causally connected region, the entropy of the string and so its length in string units are fixed and, therefore, invariant under a rescaling of the fiducial coordinates. The value of $\epsilon$ or, equivalently, $R_{\mathrm{CC}}$ is fixed as part of the definition of the state; as is the total entropy of the string state and, hence, the number of causally disconnected regions $n_{H}$. As such, all of these quantities must be independent of any rescaling of the fiducial coordinates. The same can be said of $\rho, p$, and $s$, as each of these can be strictly expressed in terms of $\epsilon$ and the coupling; see Eqs. (2)-(4). Of course, some corrections can be expected to lead to small breaking of these scale symmetries but not to any additional breaking of the translation and rotation symmetries.

It seems plausible that the same reasoning requires the various quantities to be invariant under all local Weyl transformations. This is equivalent to these quantities being invariant under all of the above transformations and, in addition, be invariant under special conformal 
transformations. Alternatively, it is expected on general grounds that scale-invariant theories are conformally invariant [33]. Either way, we will go one step further and require the fiducial coordinates to be conformally flat. ${ }^{4}$ It is unlikely that any of our conclusions are affected by this distinction.

The thermalization time scale or "scrambling time" [34] of the cosmological string state can be evaluated in a similar manner to that of BHs [11],

$$
\tau_{\text {scrambling }}=\frac{1}{H} \ln \frac{S_{\text {tot }}}{S_{H}} .
$$

The quantity $\ln \left(S_{\text {tot }} / S_{H}\right)$ will later be identified as determining the number of e-folds of inflation. Equation (13) implies that, for the total string state to reach thermal equilibrium, it has to exist for a long time compared to the light-crossing time of the causally connected regions; another manifestation of the fact that the Universe needs to be large.

A state of strings in the Hagedorn phase, for which the individual string loops are typically long (e.g., [31]), can undergo a phase transition from a bound state of hot, long strings to a state of hot radiation. This happens if the temperature decreases by a small amount below the Hagedorn temperature, leading to a reversal in entropic dominance because of $F$ changing sign. In which case, the stringy bound state will decay quickly into a gas of small string loops; that is, into a radiation-dominated phase.

It is possible that such a phase transition can be attributed to the stringy bound state being unstable to the emission of small loops; the analogue of Hawking radiation. The decay time scale $\tau_{s t}$ (as measured in fiducial string coordinates) would then be the analogue of the Page time [35], $\tau_{s t} \sim S_{H} R_{\mathrm{CC}}$, and the longest time scale in the problem from the internal microscopic perspective. The phase transition could also be induced by some coherent perturbation, which might then act on a shorter timescale. For our purposes, it is not important what brought about the phase transition, just that it occurred.

The exit from the Hagedorn phase in our model is therefore essentially the Hagedorn phase transition from a temperature above, but close to, the Hagedorn temperature, to one below it. This can be contrasted with the conjectured exit mechanism in string gas cosmology [16], which is supposed to proceed via the unwinding of strings that are initially wound around some spatial dimensions.

Let us now return to the potential issue of neglecting higher-order interactions. The strength of each additional string interaction is proportional to a factor of $N / V$, the density of potential intersection points and to an additional

\footnotetext{
${ }^{4}$ One might be concerned that the trace of the energymomentum tensor is nonvanishing. However, lacking a semiclassical Einstein equation, one cannot use properties of this tensor to constrain the symmetries of the string state.
}

factor of the coupling strength $g^{2}$. It follows that the relative strength of the $(n+2)$-string interactions in comparison to the strength of the 2-string interactions is proportional to

$$
\left(g^{2} s\right)^{n}=\epsilon^{n} \sim\left(\frac{l_{s}}{R_{\mathrm{CC}}}\right)^{n},
$$

where we have again used $s=N / V$ and Eq. (2). One can see that these are actually $\alpha^{\prime}$ corrections, being proportional to powers of $l_{s} / R_{\mathrm{CC}}$. Similarly, higher-order string-loop corrections are proportional to higher powers of $g^{2}$. Further recalling that the free energy is expressible as a power series in $s=\epsilon / g^{2}$, one can see that the higher-order interactions are thus suppressed provided that $\epsilon \ll g^{2} \ll 1$.

\section{B. Semiclassical geometric perspective: Flat slicing of de Sitter space}

As discussed in Sec. I, in looking for a semiclassical geometric description of the initial state, if one is willing to ignore the entropy and just focus on the state's mechanical aspects, then the conclusion would be that $p=-\rho$. This follows directly from the thermodynamic identity $p+\rho=$ $T s$ given that $s=0$. The resulting state can then be described by a time-independent geometry within the framework of general relativity.

Let us begin here by specifying the $p=-\rho$ geometry for a spherical volume of radius $L$. It is described by the timeindependent line element

$d s^{2}=-f(R) d T^{2}+\frac{1}{\tilde{f}(R)} d R^{2}+R^{2}\left(d \theta^{2}+\sin ^{2} \theta d \phi^{2}\right)$,

along with an energy-momentum tensor of the form $T^{\mu}{ }_{\nu}=-\rho \delta^{\mu}{ }_{\nu}$. The solution of the Einstein equations then works as

$$
\begin{aligned}
d s_{S P}^{2}= & -\left(1-\frac{R^{2}}{L^{2}}\right) d T^{2}+\frac{1}{\left(1-\frac{R^{2}}{L^{2}}\right)} d R^{2} \\
& +R^{2}\left(d \theta^{2}+\sin ^{2} \theta d \phi^{2}\right),
\end{aligned}
$$

for which

$$
\frac{1}{L^{2}}=\frac{8 \pi G}{3} \rho
$$

and $R / L \leq 1$. This solution describes the static patch of dS space, which corresponds to the interior of the

\footnotetext{
${ }^{5}$ At a first glance, $\epsilon$ and $g$ look to be the same order because the Friedmann equation $H^{2} \sim g^{2} \rho$ with $\rho \sim M_{s}^{4}$ and $H \sim \epsilon$ implies that $\epsilon^{2} \sim g^{2}$. However, when one takes numerical values seriously, it becomes clear that $H^{2}$ is suppressed significantly below this estimate; see Sec. III A.
} 
cosmological horizon at $R=L$. The energy density $\rho$ from this perspective is attributed to a cosmological constant $\Lambda=3 / L^{2}$. Notice that the geometry is spatially flat; one cannot introduce a source of spatial curvature without violating the equation of state $p=-\rho$.

If one wishes to formally extend this geometry to regions beyond the horizon, an extra leap of faith has to be taken. The formal extension to a Universe containing a large number of causally disconnected static patches - the geometry of an inflating Universe-is achieved by transforming to planar dS coordinates $r, t$ via $R=e^{H t} r$ and $e^{-2 H t}=$ $e^{-2 H T}-R^{2} H^{2}$ with $H=1 / L$. The line element then adopts the familiar form

$$
d s_{\text {planar }}^{2}=-d t^{2}+a(t)^{2} \sum_{i} d x_{i}^{2}
$$

with $a(t)=e^{H t}$ and $r^{2}=\sum_{i} x^{2}$. It should be kept in mind that the $\mathrm{dS}$ spacetime, whether in its static or planar form, is an alternative "dual" description of the initial stringy state. The string fluid is not the source in the Einstein equations that leads to a dS solution.

In spite of the apparent time dependence in the planar description, the geometry is still inherently time independent as the static-patch metric makes clear. Real time dependence, from this perspective, occurs only when the geometry is modified and some physical clock is added. It follows that the decay of the initial dS spacetime into one filled with hot radiation - which from the perspective of the late-time FRW observer corresponds to the end of inflation - has to be described by adding some external, timedependent modification to the dS geometry. Moreover, meaningful observations can only be made by the FRW observer after the state has further decayed through the properties of the matter. This will be discussed in the next subsection.

The isometries of $\mathrm{dS}$ space are important for fixing the structure of correlation functions of perturbations (see, e.g., [22,36-38]) and will also be important here for establishing the connection between the string state and its effective dS description. These are essentially the rotations and boosts of a hyperboloid in a flat spacetime with one additional dimension. When translated to planar coordinates, these include the obvious translations and rotations, along with dilatations (or scalings) and special conformal transformations. In other words, the conformal group.

\section{Comparing the microscopic and the effective semiclassical perspectives}

From the microscopic perspective, the phase transition from the initial state of strings into a state of hot radiation marks the end of inflation. Moreover, this initial state is known and sets the boundary conditions for the semiclassical evolution of gravity and matter from that point on. The matter content is also known in principle as well as the temperature, pressure, energy, and entropy densities. Alternatively, according to the semiclassical perspective, the inflationary phase of the Universe is described by an empty, time-independent half of dS space which is covered by the planar coordinates. Once inflation is over, this distinction is inconsequential.

But, as discussed in Sec. I, the score keeper for the state of the Universe is the FRW observer. This observer uses the same comoving coordinates $r$ and $t$ as for the planar dS space but can only make observations after inflation has finally ended. Meaning that the end time of inflation is the analogue of the Schwarzschild radius in the $\mathrm{BH}$ case and the inflationary past is the analogue of the $\mathrm{BH}$ interior. In short, the status of the FRW observer after inflation is quite similar to that of an observer who remains outside of a $\mathrm{BH}$. It is after the end of inflation that the FRW observer can measure the average temperature, energy density, temperature anisotropies, etc. The measurements could be done, for example, now or when the radiation of the cosmic microwave background (CMB) decoupled from other matter (or even at earlier times in principle).

From the FRW observer's perspective, the Universe starts its evolution in a thermal state, and so he would need to invent a prehistory to explain the observable Universe. He can do this in the same way that a semiclassical observer invents a description of the $\mathrm{BH}$ interior [21]. The FRW observer will conclude that the Universe was exponentially expanding at some epoch in its prethermal history, and he would then need to explain to himself how this strange era in the early Universe came about. The inflationary paradigm provides just such an explanation. Accepting this "fable," the FRW observer also has to explain the decay by smoothly matching the initial dS phase to the FRW phase. Matching in this way, he concludes that the dS phase could not really be time independent and that the "clock" keeping track of this (faux) evolution is the inflaton field. From this point of view, the inflaton does not need to correspond to any physically real field (or combination of fields) that exists in the FRW phase.

Let us reemphasize our essential point that the inflationary paradigm is an invented effective history of the Universe. What is physical and real are the results of the measurements that are made by an FRW observer after the end of inflation (i.e., after the time when the whole Universe is "created"); in particular, the measurements of the temperature anisotropies. ${ }^{6}$ If these can be predicted correctly, this is all there is to it. The rest of the theory consists of conceptualizations that can never be verified and must forever remain ambiguous.

\footnotetext{
${ }^{6}$ Note, though, that the FRW observer can only measure a portion of the postinflationary Universe at finite times. However, the whole state does become observable as $t$ increases.
} 
A remarkable fact is that the symmetries and causal structure of the string state and dS space are very similar. The string state is invariant, at the very least, under rotations, translations, and scalings (or dilatations), as is its causal connection scale $R_{\mathrm{CC}}$, which then sets the size of the horizon $1 / H$. Meanwhile, in the case of dS space, the isometries are those of the conformal group which additionally contains special conformal transformations. As explained earlier, it is likely that the string state is also invariant under the full conformal group and we proceed, for the sake of simplicity, with this as our premise. This commonality will be important for determining the correlation functions of the perturbations and showing that both perspectives lead to the same scale-invariant spectrum.

A comparison of the two alternative histories that the FRW observer can choose from is shown in Fig. 1. The semiclassical inflationary paradigm and the stringy initial state lead to the same observational results if the parameters of the inflationary model are chosen appropriately.

In summary, we proposed a novel paradigm for the early Universe: A quantum state that defines the initial state of what evolves into a semiclassical, asymptotically dS Universe and that replaces the semiclassical, singular past of the hot big-bang Universe by a quantum epoch of large but finite curvature. This quantum state of maximal entropy supplants semiclassical inflation by setting inflationary initial conditions for the hot Universe. Our idea is quite
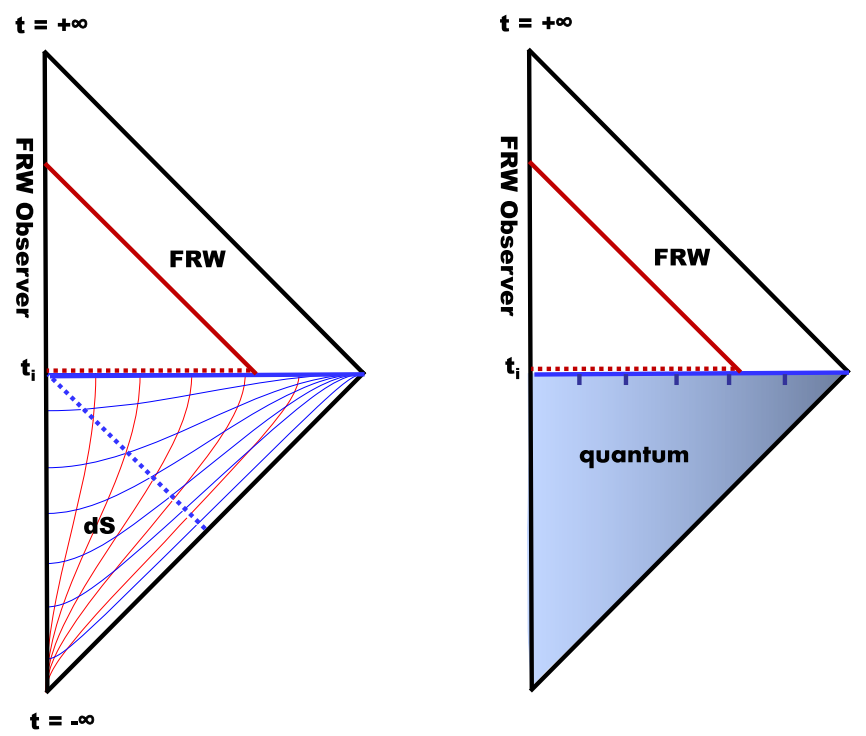

FIG. 1. Universe as viewed by the FRW observer. Shown are Penrose diagrams depicting the semiclassical interpretation (left) and the microscopic interpretation (right). Also displayed are the FRW observer's past light cone and the number of causally disconnected regions that he can see. In the lower half of the semiclassical diagram, lines of constant planar coordinates $t$ (approximately vertical) and $r$ (approximately horizontal) are shown. distinct from previous discussions about the cosmology of the Hagedorn phase of strings; for example, [16].

Our paradigm is also compatible with the swampland conjectures $[39,40]$ in an interesting way. The swampland conjectures discuss the absence of a semiclassical dS phase when the string coupling is perturbatively small and the compact volume is perturbatively large. Our model relies on having a finite string coupling and on the absence of a semiclassical description. In this sense, it complements the swampland conjectures, proposing a way in which a state that is dual to a dS space can be part of string theory. But this $\mathrm{dS}$ space is not a solution of the semiclassical string equations. It is rather an effective description, as viewed by a late time observer, of a quantum string state whose equation of state is $p=\rho$. Furthermore, an immediate consequence of our model is that $\mathrm{dS}$ gravity has a dual description in terms of a state of strings that is only metastable, as the inevitable phase transition from long, hot strings to hot, but yet cooler, radiation suggests. This also fits in nicely with the swampland conjectures.

\section{INFLATION}

As argued earlier, any invented prehistory of the Universe that is self-consistent, agrees with observations and obeys the laws of physics is as good as any other. The advantage of our microscopic description is that it unambiguously determines the parameters in the effective description of inflation and, as a by-product, resolves some of the issues that were discussed in the introductory section. Let us next explain how the effective parameters are determined. We do not discuss precise quantitative predictions here but, rather, elaborate on some of the qualitative features. A more detailed comparison to the observations will be deferred to a future article [41].

\section{A. Scale of inflation}

The energy density of the radiation just after the Hagedorn phase transition is $T_{\mathrm{Hag}}^{4}$ and recall that the Hagedorn temperature relates to the string mass as $T_{\text {Hag }}=\frac{M_{s}}{4 \pi}$. Since the Hubble parameter is given by the Friedmann equation,

$$
3 H^{2}=\frac{1}{m_{P}^{2}} T_{\text {Hag }}^{4}
$$

with $m_{P}$ denoting the reduced Planck mass $m_{P}^{2}=$ $1 /(8 \pi G)=M_{P}^{2} /(8 \pi)$, it follows that

$H=\frac{1}{\sqrt{3}(4 \pi)^{2}} \frac{M_{s}^{2}}{m_{P}} \simeq 10^{14} \mathrm{GeV}\left(\frac{M_{s}}{2.4 \times 10^{17} \mathrm{GeV}}\right)^{2}$.

We have kept track of numerical factors, both here and below, because their large values are important for ensuring the viability of the model. 
In our model the scale of inflation $H$ is expressed in terms of the fundamental string scale $M_{s}$, which is expected to be somewhat below the Planck scale $M_{P}=$ $1.2 \times 10^{19} \mathrm{GeV}$. In [42], for instance, $M_{s}$ was estimated to be of the order of $10^{17} \mathrm{GeV}$ for several types of string theories. In other scenarios it can, however, be significantly lower or, sometimes, even somewhat higher.

The entropy within a Hubble volume $S_{H}$ at the beginning of the radiation era is given by the entropy of the string state [cf. Eq. (12)],

$$
S_{H}=\pi \frac{M_{P}^{2}}{H^{2}} \simeq 10^{11}\left(\frac{2.4 \times 10^{17} \mathrm{GeV}}{M_{S}}\right)^{4},
$$

where Eq. (20) has been used in the right-most relation. This large entropy will later be related to the strength of the curvature perturbations.

Unlike for the entropies, the temperature of the radiation $T_{\text {rad }}$ is parametrically lower than the Hagedorn (string) temperature. This is because of the large number of constituent particle species, $N_{\text {species }} \gg 1$ that are expected to be in thermal equilibrium at this high a temperature, so that $T_{\text {Hag }}^{4}=N_{\text {species }} T_{\text {rad }}^{4}$.

\section{B. Duration of inflation}

The next task is to determine the duration of inflation $\tau_{\text {inflation }}$, which is a free parameter in the effective description of inflation. The accepted constraint on $\tau_{\text {inflation }}$ is that it should be long enough to explain the entropy and flatness of the observed Universe; it could, however, be much longer. Here, we will use the entropy as the physical quantity that determines the duration of inflation in the microscopic description.

Let us recall that the entropy of the stringy bound state is the total length of string in string units. We are assuming (but will later relax our assumption) that this string entropy is fully converted into radiation entropy at the phase transition which marks the ends inflation. The number of e-folds that the FRW observer has to postulate is, from his perspective, determined by the increase in volume that is required to explain the difference between the initial entropy $S_{H}$ in Eq. (21) and the total entropy of the Universe $S_{\text {tot }}=n_{H} S_{H}$,

$$
e^{3 N_{e-\text { folds }}}=\frac{V_{\text {tot }}}{H^{-3}}=n_{H}=\frac{S_{\text {tot }}}{S_{H}} .
$$

Further recall that $n_{H}$ is the number of causally disconnected patches which the string state encompasses from an external semiclassical perspective. Since the entropy is fully converted into radiation, the amount of inflation is minimal; just enough e-folds as would be necessary to "explain" the large number of causally disconnected patches. In this way, it is the value of $S_{\text {tot }}$ which determines the number of e-folds that the FRW observer has to postulate,

$$
N_{e-\text { folds }} \leq \frac{1}{3} \ln \frac{S_{\text {tot }}}{S_{H}}
$$

The right-hand side sets an upper bound because, if there were any additional entropy-generating mechanisms after inflation, it would reduce the number of e-folds that are required to explain $S_{\text {tot }}$.

In our Universe $S_{\text {tot }} \sim 10^{88}$ so that, if there are no additional sources of entropy, the requisite number of e-folds should be

$$
N_{e-\text { folds }} \sim \frac{1}{3} \ln \left(\frac{S_{\text {tot }}}{S_{H}}\right) \simeq \frac{1}{3} \ln 10^{76} \sim 60 .
$$

If we convert this number of e-folds into a dimensional duration of inflation, then

$$
\tau_{\text {inflation }}=H^{-1} N_{e-\text { folds }} \sim l_{s}\left(g^{2} S_{H}\right)^{1 / 2} \ln \left(\frac{S_{\text {tot }}}{S_{H}}\right) .
$$

Notice that the duration of inflation can be expressed strictly in terms of the parameters of the string state. The resulting time scale is, remarkably, of the same order as the scrambling time of the string state in Eq. (13), $\tau_{\text {inflation }} \sim \tau_{\text {scrambling }}$.

In spite of this last observation, let us emphasize that the number of e-folds is not directly related to any specific time dependence. It is, rather, part of the story that an FRW observer needs to tell in order to explain the fact that the initial entropy is much smaller than the final one. In addition, the FRW observer needs to introduce this artificial notion of time dependence to explain why inflation ends and how perturbations originate.

The above discussion highlights the fact that the maximal entropy state does not solve the so-called homogeneity (or smoothness or size) problem in spite of its resolution of the flatness problem. A solution of the homogeneity problem would amount to explaining why the stringy state extends over many horizon regions or, alternatively, why it is so long lived. As mentioned in the Introduction, it is likely that one needs a long phase of contraction (whether it be ultra-slow [24] or accelerated [23]) to explain the desired degree of smoothness. However, in the context of our discussion, this is a topic that is not directly relevant to the subsequent evolution of the postinflationary Universe.

\section{Perturbations}

Being strongly quantum in its nature, the initial stringy state of maximal entropy has to be fluctuating quantum mechanically. The objective here is to understand how these perturbations can be compatible with cosmological observations. 
The essential properties of the perturbations in inflation are that they "freeze" on scales larger than the horizon and are "scale invariant." The meaning of "freezing" is that their amplitudes no longer oscillate as a function of time but are (approximately) constant. The proper wave number $p=$ $|\vec{p}|$ of the perturbations when they freeze is always the horizon scale, $p=H$, but the perturbations are deemed as scale invariant when their amplitudes are independent of their comoving wave number $k=a p$ at the time of freezing. A simple way to understand both properties is to trade cosmological time $t$ for conformal time $\eta=\int \frac{d t}{a}$ so that $\eta=-1 / H a$. Then, the horizon-crossing condition for $p$ becomes $|\eta k|=1$. Hence, after crossing, the oscillating part of the perturbations in comoving coordinates $\delta \sim e^{i k \eta}$; depends on neither time nor wave number.

We will eventually reveal the origins of these two properties from the microscopic perspective while determining the amplitude of the perturbations in terms of the string scale. But even without a detailed analysis, one can anticipate these features simply because the initial string state shares with dS space the critical property of invariance under scaling transformations. Moreover, the change in the nature of the stringy perturbations across the horizon scale was already implicitly discussed in our description of the calculation of $R_{\mathrm{CC}}$ and is especially apparent from Eq. (5). The nature of these perturbations is described in detail in [43]; in particular, they are shown there to be approximately constant "outside the horizon" when $k^{2}<R_{\mathrm{CC}}^{-2}$. This constancy for perturbations that have escaped from their Hubble-sized domain may seem strange insofar as there are $n_{H}$ independent domains and, as such, variances should be suppressed by a factor of $1 / n_{H}$. However, from a quantum-mechanical perspective, it is natural to expect that the patches are not so independent but, rather, correlated through quantum entanglement. And, as is well known, quantum correlations persist over an arbitrarily long length scales.

A simple way to quantify the strength of the perturbations in the stringy description is by considering the entropy fluctuations in some region. To this end, let us consider some observable quantity $O$ in a spherical region of radius $R$ (in terms of the stringy fiducial coordinates). Then the relative strength of the quantum fluctuations of $O$ in the specified region can be defined as $\delta_{O}^{2}(R) \equiv$ $\left\langle\delta O^{2}\right\rangle_{R} /\langle O(R)\rangle^{2}$. Our main focus is on perturbations on the scale of the horizon $H \simeq 1 / R_{\mathrm{CC}}$, but we will later comment on longer length scales.

Let us next recall how the fluctuation strengths are evaluated from the free energy of the string state [11]. The essential point here is that, at fixed values of "temperature" $\epsilon$, the entropy density $s$ is essentially conjugate to the volume $V$. To see this, one can rewrite Eq. (1) as

$$
-\frac{F}{T_{\mathrm{Hag}} \epsilon}=s V-\frac{1}{2 \epsilon} g^{2} s^{2} V .
$$

The variance of the volume fluctuations at fixed temperature can now be evaluated in the standard way,

$$
(\delta V)_{\epsilon}^{2}=\frac{\partial^{2}\left(F / T_{\mathrm{Hag}} \epsilon\right)}{\partial s^{2}}=\frac{g^{2} V}{\epsilon} .
$$

Substituting for $\epsilon$ from Eq. (2) and dividing by $V^{2}$, we now obtain

$$
\delta_{V}^{2}(R)=\frac{1}{S(R)},
$$

for some region of size $R$.

The conjugacy of $s$ and $V$ ensures that their respective fluctuations have the same relative strength, so that

$$
\delta_{s}^{2}\left(R_{\mathrm{CC}}\right) \simeq \frac{1}{S\left(R_{\mathrm{CC}}\right)}
$$

And, since $s$ and $\rho$ differ only by a single factor of (constant) $\epsilon$,

$$
\delta_{\rho}^{2}\left(R_{\mathrm{CC}}\right) \simeq \frac{1}{S\left(R_{\mathrm{CC}}\right)}=\frac{1}{\pi} \frac{R_{\mathrm{CC}}^{2}}{M_{P}^{2}}
$$

or, in more conventional notation,

$$
\delta_{\rho}^{2}(H) \simeq \frac{1}{\pi} \frac{H^{2}}{M_{P}^{2}},
$$

where the argument $H$ has been used as short hand for a Hubble-sized length scale $R_{\mathrm{CC}} \simeq 1 / H$. The standard inflationary result scales in the same way, as $P_{\rho}(k)=\frac{2}{\pi} \frac{H^{2}}{M_{P}^{2}}$ is the closely related power spectrum for the tensor perturbations [44]. As already mentioned, we will conduct a precise quantitative comparison between our results and those of the inflationary paradigm in a separate publication [41].

From the semiclassical perspective, Eq. (31) quantifies the strength of the tensor curvature perturbations rather than the scalar curvature perturbations, which will be discussed next. These tensor perturbations are, therefore, the most direct link between the stringy description and the effective model of inflation. Using either Eq. (20) or (21), one can estimate their strength in terms of the value of the string scale $M_{s}$.

A less direct link is provided by the value of the gaugeinvariant scalar curvature perturbation $\zeta$ [44]. We will rely on the relationship between $\zeta$ and the perturbation in the

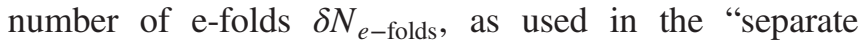
Universe" approach and the $\delta N$ formalism [45,46] for the calculation of superhorizon perturbations,

$$
\zeta=\delta N_{e-\text { folds }}
$$


This identity will be used here as the defining relation of the gauge-invariant scalar perturbation in the maximal entropy state,

$$
\zeta_{s t} \equiv \delta N_{e-\text { folds }}
$$

As shown below, the value of $\delta N_{e-\text { folds }}$ can be expressed in terms of quantities that are well defined for the maximalentropy state.

The number of e-folds in the maximal entropy state can be expressed as in Eq. (22),

$$
N_{e-\text { folds }}=\int d t \frac{1}{3} \partial_{t} \ln V \simeq \int d t \partial_{t} \ln S,
$$

from which it follows that

$$
\left.\delta N_{e-\text { folds }} \simeq \int d t \partial_{t} \frac{\delta S_{H}}{S_{H}} \simeq \int d t H \frac{\delta s}{s}\right|_{H} .
$$

It can then be concluded that

$$
\zeta_{s t}=\left.\int d t H \frac{\delta s}{s}\right|_{H} .
$$

It is also noteworthy that

$$
\dot{\zeta}_{s t}=\left.H \frac{\delta s}{s}\right|_{H},
$$

similar to the case in which the perturbations are nonadiabatic [44,47].

It should be emphasized that the above time derivatives refer to the time coordinate $t_{s t}$ of the string state's fiducial (conformally flat) system and not that of the planar dS space. However, as both descriptions share the property of conformal invariance, we have the freedom to match their respective coordinate systems with one another and with those of the FRW observer at the surface $r=H^{-1}$ (equivalently, at $r_{s t}=R_{\mathrm{CC}}$ ). In other words, once at or outside the horizon, all relevant observers can be assigned a common definition of time, and we can subsequently adopt the coordinate $t$ of planar dS without any loss of generality. Nonetheless, as explained below (also see [22]), any time dependence in the calculation is something of a red herring and so one's particular choice of time coordinate is almost besides the point.

The magnitude of the scalar curvature perturbation at the horizon can be evaluated as follows:

$$
\begin{aligned}
\left\langle\zeta_{s t}^{2}\right\rangle_{H} & =\int d t^{\prime} \int d t^{\prime \prime} H^{2}\left\langle\frac{\delta s}{s}\left(t^{\prime}\right) \frac{\delta s}{s}\left(t^{\prime \prime}\right)\right\rangle_{H} \\
& \simeq H \int d t \delta_{s}^{2}(H) \simeq H \int d t \delta_{\rho}^{2}(H) .
\end{aligned}
$$

Here, $\left\langle\frac{\delta s}{s}\left(t^{\prime}\right) \frac{\delta s}{s}\left(t^{\prime \prime}\right)\right\rangle_{H} \sim H^{-1} \delta\left(t^{\prime}-t^{\prime \prime}\right)$ has been used. The reason that a delta function appears here is basically the same reason that it appears in the standard inflationary calculations. The horizon-crossing constraint-or its casual-connection analogue-means that at times such that $H\left|t^{\prime}-t^{\prime \prime}\right|>1$, the perturbations $\zeta_{s t}\left(t^{\prime}\right)$ and $\zeta_{s t}\left(t^{\prime \prime}\right)$ are uncorrelated. From Eq. (38), one can observe that $\zeta_{s t}$ evolves outside the horizon, again, as in the case of nonadiabatic perturbations.

The remaining integration over time can be interpreted as the time to probe the total length of string, which amounts to an integration over the number $n_{H}$ of independent domains. To see this, one can apply $H d t \simeq d N_{e-\text { folds }}$ to obtain

$$
\left\langle\zeta_{s t}^{2}\right\rangle_{H} \sim \int d N_{e-\text { fold }} \delta_{\rho}^{2}(H) \sim \int d \ln n_{H} \delta_{\rho}^{2}(H),
$$

where Eq. (22) has been used. The last equality also makes it clear that Eq. (39) is expressed entirely in terms of the microscopic description.

If one assumes that $\delta_{\rho}^{2}(H)$ remains approximately constant over the $n_{H}$ disconnected regions, the above integral simplifies to

$$
\left\langle\zeta_{s t}^{2}\right\rangle_{H} \sim N_{e-\text { folds }} \delta_{\rho}^{2}(H) .
$$

The factor of $N_{e-\text { folds }}$ represents an enhancement factor of the scalar perturbation over the tensor perturbations. Indeed, the tensor-to-scalar ratio $r$ is given by

$$
r=\frac{\delta_{\rho}^{2}(H)}{\left\langle\zeta_{s t}^{2}\right\rangle_{H}} \simeq \frac{1}{N_{e-\text { folds }}} .
$$

The calculation of the scalar perturbations and the resulting enhancement does not depend explicitly on any deviations from scale invariance. The quantity $\zeta_{s t}$ therefore shares the properties of the standard gauge-invariant scalar perturbation $\zeta$-it is the physical clock.

The calculation of the $\zeta_{s t}$ correlation function can, as alluded to, also be done in a way that eliminates the time dependence altogether. Let us reconsider the double time integral in Eq. (38) and rewrite each integral in terms of conformal time, $\int d t \rightarrow \int a d \eta$. Applying the derivative of the comoving form of horizon-crossing constraint $k d \eta+\eta d k=0$, one then has $\int a d \eta \rightarrow-\int \frac{\eta}{k} a d k=$ $\int \frac{1}{k H} d k$. If one also approximates the time derivatives by $H$, the double time integral in Eq. (38) becomes a double integral in $k$-space,

$$
\left\langle\zeta_{s t}^{2}\right\rangle_{H}=\int d\left(\ln k^{\prime}\right) \int d\left(\ln k^{\prime \prime}\right)\left\langle\zeta_{s t}\left(k^{\prime}\right) \zeta_{s t}\left(k^{\prime \prime}\right)\right\rangle_{H} .
$$

Further imposing the delta function resulting from momentum conservation, we obtain 


$$
\left\langle\zeta_{s t}^{2}\right\rangle_{H} \sim \int d(\ln k)\left\langle\zeta_{s t}^{2}(k)\right\rangle_{H} \simeq \int d(\ln k) P_{\zeta_{s t}}(k),
$$

where the right-most equality follows from the standard relation between a two-point correlation function in position space and its associated power spectrum. From Eq. (38), it can then be deduced that

$$
\left\langle\zeta_{s t}^{2}\right\rangle_{H} \simeq N_{e-\text { folds }} \int d(\ln k) P_{\rho}(k)
$$

This makes clear that both the time-dependence of the scalar perturbations and their scale invariance can be presented in a way that can be viewed purely in terms of the Fourier modes of the string state.

By working within the microscopic picture, we did not need to introduce nor rely on any time dependence. Notice also that the enhancement of the scalar perturbations with respect to the tensor perturbations was arrived at in a way that does not depend explicitly on any deviations from a scale invariant state. The tensor fluctuations, which represent the fundamental quantity from this perspective, depend only on the thermodynamic properties of the initial string state. Meanwhile, the enhancement factor for the scalar modes can be attributed to the largeness of the initial state rather than to a contrived period of dynamical evolution. Additional deviations could arise from subleading terms and departures from exact equilibrium; see below. In our model, the deviation of the spectrum of scalar perturbations from scale invariance does not fix $r$, rather the smallness of $r$ is related to the fact that the initial state is large and thus has a large entropy. This can be contrasted with the predictions of string gas cosmology and holographic cosmology, as in both models $r$ vanishes for an exactly scale invariant scalar spectrum $[18,20]$.

Now what happens on scales larger than the horizon? The answer is simple: essentially the same as in the standard inflationary picture. This is because both the freezing of the amplitudes (or their growth) and the scale invariance of the spectrum are direct consequences of the scaling symmetry of the dS space and the form of the perturbation equation. As we have shown, the corresponding symmetries and perturbation equations of the microscopic string state have the same relevant properties as those in dS space. The scaling symmetry ensures, in particular, that the position of the horizon scale and the causal-connection scale $R_{\mathrm{CC}}$ are invariant features of $\mathrm{dS}$ space and the microscopic string state, respectively.

More formally and as reviewed recently in [22,48], given a two-point function $\left\langle\delta \phi^{2}\right\rangle$ in position space that is invariant under dilatations $x^{a} \frac{d}{d x_{a}}$, its two-point function in Fourier space (i.e., its power spectrum) has to have the following form at all trans-Hubble scales:

$$
P_{\phi}(k)=\frac{k^{3}}{2 \pi^{2}}\langle\delta \phi(\vec{k}) \delta \phi(-\vec{k})\rangle=C_{\phi}(H),
$$

where $C_{\phi}$ is a function of $H$ only. As the tensor and scalar perturbations inherit the same set of symmetries from the string state as they do from dS space, all that is left is to fix the value of the $C$ 's, which we know to be $C_{\rho}(H) \simeq H^{2} / M_{P}^{2}$ from Eq. (31) and then $C_{\zeta_{s t}}(H) \simeq N_{e \text {-folds }} H^{2} / M_{P}^{2}$ due to the enhancement.

Let us now discuss possible deviations from Gaussianity and scale invariance. In the approximation that the free energy of the string state is quadratic and the state is in equilibrium, the fluctuations of the various quantities are strictly Gaussian.

Deviations from Gaussianity then depend on the relative strength of the higher-order string interactions. As discussed in Sec. II, the relative strength of the $(n+2)$-string interactions in comparison to the strength of the 2-string interactions scales as $\left(\frac{g^{2} N}{V}\right)^{n}=\epsilon^{n}=\left(\frac{H}{M_{s}}\right)^{n}$. These are, as already mentioned, highly suppressed $\alpha^{\prime}$ corrections, which indicates that the perturbations are approximately Gaussian. Higher-order string-loop corrections (and combinations thereof) are also possible but these would of course be suppressed by additional factors of $g^{2}$. Deviations from strict scale invariance are more subtle and will be discussed in more detail in [41].

\section{SUMMARY AND CONCLUSION}

We have presented a novel paradigm for the early Universe which resolves the initial singularity problem of inflation and yet can reproduce nontrivial qualitative features of the spectrum of inflationary perturbations. Our proposal is that the initial state is a thermal state of closed, interacting, fundamental strings at a temperature which is just above but parametrically close to the Hagedorn temperature. We included a complete description of the initial state for given values of the total entropy (or equivalently, the total volume), the Hagedorn temperature, the temperature of the state and the string coupling. Our proposed state thus provides a microscopic model of inflation.

We showed that, qualitatively, all the essential features of inflation are reproduced. The overall scale of the perturbations and the ratio of scalar-to-tensor perturbations are, surprisingly, qualitatively in agreement with observations. The first surprise is that a string-scale energy density gives a Hubble scale that is a factor of about $10^{-5}$ lower than the Planck scale in spite of the fact that the two scales, string and Planck, are quite close. The second, even bigger surprise is that the ratio of scalar-to-tensor perturbations is parametrically small, scaling as the inverse of the total number of e-folds. In previous attempts to replace semiclassical inflation by an initial state, this ratio was generally found to be of order one. Achieving a hierarchy between the scalar and tensor perturbations is considered as the main 
obstacle for such a replacement and, as far as we know, ours is a first example of such a hierarchy. Furthermore, the qualitative prediction of a high scale and a minimally acceptable tensor-to-scalar ratio means that our model predicts that the tensor perturbations are observable.

Our model is premised on a simple idea, that the resolution of a spacelike singularity-whether it be at the center of a $\mathrm{BH}$ or as a precursor to the big bangrequires large deviations from semiclassical physics over horizon-sized scales. Large deviations in this sense means that the region of spacetime lacks a description in terms of a semiclassical geometry. Such a situation occurs for strongly coupled states of matter, which are synonymous with states of extremely large entropy. The Hagedorn phase of closed strings is one notable example of just such a state.

From our perspective, any description of the early Universe up to the end of the inflationary phase is as valid as any other, provided that it is self-consistent, agrees with observations and obeys the known laws of physics. This puts the late-time or FRW observer on equal footing with the exterior observer in the $\mathrm{BH}$ case, as both can only "make up stories" about the early Universe and the BH interior respectively. Our story is one of a cosmological picture that is devoid of singularities but deprived of a semiclassical geometry at early times. On the other hand, the observer who is determined to maintain a semiclassical description of the inflationary period will take our picture from one extreme, maximal entropy and maximally positive pressure, to another, zero entropy and maximally negative pressure. The later extreme being the more conventional dS description of inflation.

What is then to be gained from our stringy description of the early Universe? The answer being that our model fixes the parameters of inflation in terms of two fundamental quantities, the string mass and the string coupling. As an added bonus, this model can be connected to $\mathrm{BH}$ physics, for which the same two parameters can also be probed. The only other input is the minimal number of e-folds that is needed to explain the size of the Universe. Our description also manages to evade some of the usual conceptual issues haunting inflation, such as the identity of the inflaton and the self-consistency problems, such as eternal inflation, that ensue from that perspective. Furthermore, the inflaton models tend to have many adjustable parameters and a myriad of moving parts. In short, the lack of opportunities for "tweaking" means that our framework is predictive and will be much easier to substantiate (or falsify). The relatively small suppression of the tensor perturbations with respect to the scalar perturbations suggests that they could be discovered sooner than later. A sequel to the current article which includes a substantially more quantitative analysis of the observable consequences of our model will be presented in due course [41].

\section{ACKNOWLEDGMENTS}

We would like to thank Marko Simonovic, Paul Steinhardt, and Gabriele Veneziano for discussions and in particular Toni Riotto, for fruitful discussions and useful suggestions. The research of R. B. was supported by the Israel Science Foundation Grant No. 1294/16. The research of A.J.M.M. received support from an NRF Incentive Funding Grant 85353 and a Rhodes University Discretionary Grant No. RD51/2018. A. J. M. M. thanks Ben Gurion University for their hospitality during his visit.
[1] A. D. Linde, Particle physics and inflationary cosmology, Contemp. Concepts Phys. 5, 1 (1990).

[2] A. H. Guth, The Inflationary Universe: The Quest for a New Theory of Cosmic Origins (Addison-Wesley, Reading, USA, 1997).

[3] A. Ijjas and P. J. Steinhardt, Implications of Planck2015 for inflationary, ekpyrotic and anamorphic bouncing cosmologies, Classical Quantum Gravity 33, 044001 (2016).

[4] R. Brandenberger, Inflationary Cosmology: Progress and Problems, NAO Colloquium, 2015, http://colloquium.bao .ac.cn/sites/default/files/ppt-NO.028_2015.pdf.

[5] D. Chowdhury, J. Martin, C. Ringeval, and V. Vennin, Inflation after Planck: Judgment Day, Phys. Rev. D 100, 083537 (2019); J. Martin, Cosmic inflation: Trick or treat?, arXiv:1902.05286.

[6] A. Borde and A. Vilenkin, Eternal Inflation and the Initial Singularity, Phys. Rev. Lett. 72, 3305 (1994).
[7] A. Borde, A. H. Guth, and A. Vilenkin, Inflationary SpaceTimes are Incomplete in Past Directions, Phys. Rev. Lett. 90, 151301 (2003).

[8] S. W. Hawking, Nature of space and time, arXiv:hep-th/ 9409195.

[9] R. Brustein and A. J. M. Medved, Resisting collapse: How matter inside a black hole can withstand gravity, Phys. Rev. D 99, 064019 (2019).

[10] R. Brustein and A. J. M. Medved, Black holes as collapsed polymers, Fortsch. Phys. 65, 1600114 (2017).

[11] R. Brustein and A. J. M. Medved, Emergent horizon, Hawking radiation and chaos in the collapsed polymer model of a black hole, Fortsch. Phys. 65, 1600116 (2017).

[12] R. Brustein and G. Veneziano, A Causal Entropy Bound, Phys. Rev. Lett. 84, 5695 (2000).

[13] G. Veneziano, Entropy bounds and string cosmology, arXiv: hep-th/9907012. 
[14] R. H. Brandenberger and C. Vafa, Superstrings in the early universe, Nucl. Phys. B316, 391 (1989).

[15] A. Nayeri, R. H. Brandenberger, and C. Vafa, Producing a Scale-Invariant Spectrum of Perturbations in a Hagedorn Phase of String Cosmology, Phys. Rev. Lett. 97, 021302 (2006).

[16] R. H. Brandenberger, String Cosmology, edited by J. Erdmenger (Wiley, New York, 2009), p. 193-230.

[17] R. H. Brandenberger, A. Nayeri, S. P. Patil, and C. Vafa, Tensor Modes from a Primordial Hagedorn Phase of String Cosmology, Phys. Rev. Lett. 98, 231302 (2007).

[18] R. H. Brandenberger, A. Nayeri, and S. P. Patil, Closed string thermodynamics and a blue tensor spectrum, Phys. Rev. D 90, 067301 (2014).

[19] T. Banks and W. Fischler, The holographic spacetime model of cosmology, Int. J. Mod. Phys. D 27, 1846005 (2018).

[20] T. Banks and W. Fischler, Holographic Inflation Revised (Cambridge University Press, Cambridge, England, 2017).

[21] R. Brustein and A. J. M. Medved, Non-singular black holes interiors need physics beyond the standard model, Fortsch. Phys. 67, 1900058 (2019).

[22] N. Arkani-Hamed, D. Baumann, H. Lee, and G. L. Pimentel, The cosmological bootstrap: Inflationary correlators from symmetries and singularities, J. High Energy Phys. 04 (2020) 105.

[23] M. Gasperini and G. Veneziano, The Pre-big bang scenario in string cosmology, Phys. Rep. 373, 1 (2003).

[24] J. Khoury, B. A. Ovrut, P. J. Steinhardt, and N. Turok, The ekpyrotic universe: Colliding branes and the origin of the hot big bang, Phys. Rev. D 64, 123522 (2001).

[25] T. Damour and G. Veneziano, Selfgravitating fundamental strings and black holes, Nucl. Phys. B568, 93 (2000).

[26] S. Fubini and G. Veneziano, Level structure of dual resonance models, Nuovo Cimento 64A, 1640 (1969).

[27] J. J. Atick and E. Witten, The Hagedorn transition and the number of degrees of freedom in string theory, Nucl. Phys. B310, 291 (1988).

[28] N. Deo, S. Jain, and C.-I. Tan, String distributions above Hagedorn energy density, Phys. Rev. D 40, 2626 (1989).

[29] P. G. De Gennes, Scaling Concepts in Polymer Physics (Cornell University, Ithaca, New York, 1979).

[30] G. W. Gibbons and S. W. Hawking, Cosmological event horizons, thermodynamics, and particle creation, Phys. Rev. D 15, 2738 (1977).

[31] D. A. Lowe and L. Thorlacius, Hot string soup: Thermodynamics of strings near the Hagedorn transition, Phys. Rev. D 51, 665 (1995).
[32] G. T. Horowitz and J. Polchinski, Selfgravitating fundamental strings, Phys. Rev. D 57, 2557 (1998).

[33] Y. Nakayama, Scale invariance vs conformal invariance, Phys. Rep. 569, 1 (2015).

[34] P. Hayden and J. Preskill, Black holes as mirrors: Quantum information in random subsystems, J. High Energy Phys. 09 (2007) 120.

[35] D. N. Page, Average Entropy of a Subsystem, Phys. Rev. Lett. 71, 1291 (1993); Information in Black Hole Radiation, Phys. Rev. Lett. 71, 3743 (1993).

[36] J. M. Maldacena and G. L. Pimentel, On graviton nonGaussianities during inflation, J. High Energy Phys. 09 (2011) 045.

[37] A. Bzowski, P. McFadden, and K. Skenderis, Implications of conformal invariance in momentum space, J. High Energy Phys. 03 (2014) 111.

[38] N. Kundu, A. Shukla, and S. P. Trivedi, Constraints from conformal symmetry on the three point scalar correlator in inflation, J. High Energy Phys. 04 (2015) 061.

[39] G. Obied, H. Ooguri, L. Spodyneiko, and C. Vafa, De Sitter space and the swampland, arXiv:1806.08362.

[40] E. Palti, The swampland: Introduction and review, Fortsch. Phys. 67, 1900037 (2019)

[41] R. Brustein and A. J. M. Medved, A correspondence between strings in the Hagedorn phase and asymptotically de Sitter space, arXiv:2005.09321.

[42] R. Brustein, S. P. De Alwis, and E. G. Novak, Inflationary cosmology in the central region of string/M theory moduli space, Phys. Rev. D 68, 023517 (2003).

[43] R. Brustein, M. Gasperini, and G. Veneziano, Duality in cosmological perturbation theory, Phys. Lett. B 431, 277 (1998).

[44] V. F. Mukhanov, H. A. Feldman, and R. H. Brandenberger, Theory of cosmological perturbations. Part 1. Classical perturbations. Part 2. Quantum theory of perturbations. Part 3. Extensions, Phys. Rep. 215, 203 (1992).

[45] M. Sasaki and E. D. Stewart, A General analytic formula for the spectral index of the density perturbations produced during inflation, Prog. Theor. Phys. 95, 71 (1996).

[46] D. Wands, K. A. Malik, D. H. Lyth, and A. R. Liddle, A new approach to the evolution of cosmological perturbations on large scales, Phys. Rev. D 62, 043527 (2000).

[47] D. H. Lyth, C. Ungarelli, and D. Wands, The primordial density perturbation in the curvaton scenario, Phys. Rev. D 67, 023503 (2003).

[48] A. Riotto, Inflation and the Theory of Cosmological Perturbations, GGI lectures on the theory of fundamental interactions, 2018. 